\title{
Antiproliferative activity of Stokesia laevis ethanolic extract in combination with several food-related bioactive compounds; in vitro (Caco2) and in silico docking (TNKS1) studies
}

\author{
Georgeta Neagu ${ }^{1}$, Amalia Stefaniu ${ }^{1}$, Adrian Albulescu ${ }^{1,2}$, Lucia Pintilie ${ }^{1}$, Lucia Camelia Pirvu ${ }^{1, *}$ \\ 1 National Institute for Chemical-Pharmaceutical Research and Development, ICCF, 112 Vitan Av., \\ 031299 Bucharest, Romania; getabios@yahoo.com (G.N.); astefaniu@gmail.com (AS); \\ rockady2@gmail.com (A.A.); lucia.pintilie@gmail.com (L.P.); lucia.pirvu@yahoo.com (L.C.P.) \\ 2 "Stefan S. Nicolau" Institute of Virology, 285 Mihai Bravu Av., 030304, Bucharest, Romania; \\ rockady2@gmail.com (A.A); \\ * Correspondence: lucia.pirvu@yahoo.com;
}

Featured Application: Developing new natural or combined drugs with antitumor activity.

\begin{abstract}
In this study, five polyphenol compounds (reference substances, ref.) were added to Stokesia laevis (Slae26) ethanolic extract $(5 \mathrm{mg}$ GAE / $\mathrm{mL}$ ) in order to test their antiproliferative effects on human colon tumor cell line Caco-2 (ATCC-HTB-37). The five phenolics (ref.) are luteolin-7-O-glucoside (orientin), luteolin-8-C-glucoside (cinnaroside), caffeic acid, gentisic acid and para-aminobenzoic acid (PABA), and they were combined in ratio 1:1 between the active compounds in samples (GAE : ref.). Results indicated certain antiproliferative effects of Slae26 $\left(\mathrm{IC}_{50}=36 \mu \mathrm{g}\right.$ GAE$/ \mathrm{mL}$ sample), of the five reference compounds, but mainly of Slae26 combinations with the five phenolics tested (ref.). Punctually, there were obtained IC 50 values around $5 \mu \mathrm{g} / \mathrm{mL}$ sample, signifying real therapeutic usefulness and antitumor potency of Slae26 combinations with the five food bioactive compounds tested. In silico docking studies provided potential therapeutic agents to inhibit the catalytic domain of human tankyrase 1 (TNKS1) in the series of food bioactive compounds tested, and revealed luteolin-7-O-glucoside high effectiveness.
\end{abstract}

Keywords: food bioactive compounds; stokes aster ethanolic extracts; health effects; antiproliferative activity; Caco2 cells; docking; human tankyrase 1.

\section{Introduction}

Green plants contain an impressive number of chemical compounds (estimated at several thousand distinct molecules), which are classified as either derivatives of primary metabolism or derivatives of the secondary metabolism [1]. However, some recent studies show that there is no absolute delimitation between the two major processes in the plant [2]. For example, it was proved that under certain conditions, the secondary metabolism can "borrow" enzymes from the primary metabolism [3]; also, an adaptation of the synthesis of secondary metabolites depending on the punctual need of a plant (e.g., in the process of acclimatization or domestication of plants [4]) has been proved, which led to the conclusion of a greater flexibility of the specialized metabolism in plants [5].

Broadly, it is accepted that the derivatives of primary metabolism (namely central carbon metabolism) are essentially carbohydrates that directly contribute to the growth and development of the plant having a plastic or structural role, along with compounds with functional and metabolic role, such as proteins (enzymes), lipids, nucleotides, amino 
acids, organic acids, phytosterols and minerals. Secondary metabolism derivatives refer to compounds that do not have a direct function in basic metabolism of plants and are differentiate distributed between taxonomic groups in the plant kingdom [6] but originate from the processes of the primary metabolism. This category includes more than 50 subclasses of phytocompounds, such as: polyphenols compounds (phenolic acids, flavonoids, coumarins, lignans, quinones) and other phenolics including lignin ester (a complex of esterified phenolic acids that participate in the formation of plant cell walls providing impermeability of the structure), volatile oils, terpenoids (bitter substances), triterpene acids and saponosides, alkaloids, lectins, phytoalexins, etc. [1-2]. Secondary metabolites have a very important adaptive role as they ensure the interaction between the plant and the environment, thus protecting the plant against physical, chemical, and biological agents, as well as against herbivorous assault, while ensuring the multiplication of the species through compounds with an attractive role (e.g., pigments, perfumes, dyes, etc.), or are pollinating or allelopathic agents and thus influence the competition between plant species [7-9].

In this context, food-related bioactive compounds are not only significant in their number, but also very complex molecules, their complexity being due to the wide range of chemical structures and their association into macromolecular and polymeric structures. This complexity, together with the uniqueness of the chemical composition in plant species, is reflected both, in the nutritional value of the species, and numerous pharmacological activities and benefits for human health. The reality of benefits of plant compounds for human health support is proved by studies reporting that "about $30 \%$ of the worldwide sales of drugs are based on natural products [10]. Thus, it was counted that "over $60 \%$ of approved drugs are derived from natural compounds [11], and among 185 small molecules used in the treatment of cancer, 33.5\% belong to natural compounds [12]. Data also shown that pharmaceutical companies spend approximately US \$350 million to develop a new drug [13]. Punctually, from the total number of 74 areas of disease at humans, 27 areas are in the situation of total lack of $d r u g s$ derived from natural products [12]; the indications related to the disease areas that do not have at least one drug based on unaltered natural compound (N), botanical drug (NB) or natural product derivative (ND) are the following: anesthetic, antianginal, antiemetic, antihistamine, antimigraine, antipsychotic, anxiolytic, chelator, cystic fibrosis, diuretic, erythropoiesis, gastroprokinetic, hematopoiesis, hemophilia, hemostatic, hypercholesterolemia, hyperphosphatemia, hypnotic, idiopathic thrombocytopenia, infertility at women, male sexual dysfunction, neuroleptic, schizophrenia, urinary incontinence, and pulmonary arterial hypertension and chronic obstruction pulmonary disease.

Furthermore, plant compounds druggability studies proved that the first four rules of Lipinski [14] do not apply to the natural products or other molecules that are the subject of an active transport system [15-17].

In terms of food-related secondary metabolites in plants, studies [18] on the common western type of food indicated that most active compounds with certain health benefits for humans come from the cocoa and tea products, berries (black chokeberry, blueberries, blackberries, red raspberries and strawberries) and non-berries fruits (citrus, apples, black currants, plums, sweet cherries), red wine, some vegetables (artichokes, chicory, red onions, spinach), nuts (hazelnuts, pecans, almonds, walnuts), soy products, olive oil and spices (ginger, turmeric, pepper, peppermint, star anise, basil, cloves, etc.). If also considering the frequency and amount commonly used in daily diet, it was estimated that chlorogenic acid isomers and its metabolites (e.g., caffeic acid, ferulic and isoferulic acids) and some flavan-3-ols compounds (e.g., catechins and epi(gallo)catechin gallates derivates), are the dominant food-related bioactive compounds [18].

Furthermore, the bioavailability of secondary metabolites in their natural form in plants (glycosides and esters) is generally estimated at less than 5\% [19-20]. Then, human 
health benefits of food-related bioactive compounds especially come from their direct effect or direct contact with the components of the digestive system; the compounds which pass through the mucous lining of the stomach and intestine only, termed compounds with bioavailability in humans, can act at the level of organs, or can have a systemic effect. Also considering the inter-individual variability in humans [21-22], it results that the benefits of food-related bioactive compounds is mostly played by the individual microbiota, which in fact metabolizes most of the ingested plant secondary metabolites.

Yet, clinical studies along the time concluded that the aglycones of plant secondary metabolites generally have the condition of good bioavailability in humans, and some glycosides and esters use some specialized enzymes from intestine, therefore they can achieve high bioavailability in humas (over $40 \%$ ); they are quercetin derivates from onions (quercetin 4'-O-beta-D-glucopyranoside namely spiraeoside) and apples (quercetin 3-O-beta-D-glucoside namely isoquercitrin), and procyanidins and epigallocatechin gallates from green tea, wine and berries. Also, ferulic acid was proved to cross both, the stomach mucous layer and the blood-brain barrier, due to its combination of low molecular weight and low polarity induced to the methyl group and naringenin flavanone demonstrated the ability to influence the bioavailability of other natural and synthetic compounds [19-20]; moreover, caffeic, chlorogenic, ferulic and ellagic acids were proved to be the subject of the active transport in humans [21-23].

Besides these, food-related bioactive compounds must pass the bio-accessibility step [24], prior to being the subject of bioavailability and absorption. Defined as the fraction of a compound which is released from the food matrix in gastrointestinal lumen, the bio-accessibility of a compound is influenced by numerous physical, chemical, and biological (enzymatic processes) factors; e.g., the food texture and the type of matrix, mastication grade, temperature and $p \mathrm{H}$, but also the functioning of digestive enzymes and bile secretion, caloric content and the volume of food matrix, and other food processing operations such as fermentation, coagulation, partial hydrolysis, etc. [24]. Consequently, since the chemical pharmaceutical industry can improve the efficacy of secondary metabolites from plants by proper combination of ingredients and an appropriate formulation (e.g., by microencapsulation, use of liposomes, microemulsion into fats, etc.), the functional ingredients food industry is disadvantaged exactly by the lack of predictability of bio-accessibility and bioavailability of food-related bioactive compounds [24-25].

However, there is clear evidence of the efficacy of plant compounds, both in the prevention and effective treatment of many human diseases [26-28]. A problem that remains to be solved is that of the active dose, even in the case of the most active plant derived products; it involves the extracts/compounds/plant derived products with an IC 50 smaller than $50 \mu \mathrm{g}$ active compounds per $1 \mathrm{~mL}$ sample. This disadvantage of excessively high doses of plant products is even more challenges in the case of samples with certain antitumor activity and without toxic effects on the normal cells, the more so as studies show that the whole (unrefined) vegetal extracts are more active than the selective extracts or single compounds [29-32]; the higher efficacy of unrefined extracts is explained by the complexity (numerical and structural) of compounds in vegetal extracts curing the damaged tissue or overwhelming numerous defense reactions of target tissue.

The goal of this paper was to study the antiproliferative activity of an ethanolic extract from Stokesia laevis (Slae26) and its combinations with several polyphenols compounds (reference substances, ref.) on the human colon tumor cell line Caco-2 (ATCC-HTB-37), in an attempt to increase the biological activity of Slae26 by concentrating it in own active compounds and other food-related bioactive compounds with high bioavailability and additionally benefits in cancer fight at humans. Reference compounds selected for the study were: luteolin 8-C-glucoside (orientin), luteolin 7-O-glucoside (cinnaroside), caffeic acid, gentisic acid and para-aminobenzoic acid 
(PABA). Also, molecular docking simulation on the five phenolics (ref.), upon the target human tankyrase 1 (TNKS1), involved in colon cancer development, have been done.

\section{Materials and Methods}

Plant material description: The vegetal raw material (Stokesia laevis L. - herba et flores) was acquired from a specialized plant distributor in Romania, and its taxonomic identity was confirmed by the botanist's team of National Institute of Chemical-Pharmaceutical R\&D, Bucharest, Romania. The fresh flowering plant was cut (the aerial part), shade dried and ground to a medium-size powder product. Voucher specimens, the dried plant, and the powder product (Slae), are deposited in the ICCF Plant Material Storing Room.

Chemicals, reagents and references: Chemicals (e.g., sodium carbonate), reagents (e.g., Folin-Ciocalteau, Natural Product-NP/PEG), solvents (e.g., ethanol, ethyl acetate, formic acid and glacial acetic acid), the reference compounds for quantitative and qualitative studies namely rutin ( $\mathrm{min} .95 \%)$, quercetin-3-O-rhamnoside $(>90 \%)$, luteolin $(>98 \%)$, luteolin-7-O-glucoside( $>98 \%)$, luteolin-8-C-glucoside (>97\%), caffeic acid $(99 \%)$, chlorogenic acid (>95\%) and gallic acid (95\%), and the cell culture reagents used, Dulbecco's Modified Essential Media (DMEM) and Foetal Bovine Serum (FBS), were purchased from Sigma-Aldrich Company Distributor in Romania.

Plant extracts preparation: Fifty (50) grams of stokes aster plant powder was extracted on the water bath (in a glass device of $2 \mathrm{~L}$ ) with $1000 \mathrm{~mL}$ of $70 \%(\mathrm{v} / \mathrm{v})$ ethanol, for one hour at the boiling temperature. The filtered ethanolic extract (Slae) in a volume of $745 \mathrm{~mL}$ was analyzed for qualitative (polyphenols profile) and quantitative (total phenols content) aspects. An aliquot of $250 \mathrm{~mL}$ of ethanol extract with the content of 0.61 mg GAE per $1 \mathrm{~mL}$ sample was further concentrated at sicc product (residue) and the sicc product was solved into $30.5 \mathrm{~mL}$ ethanol $40 \%(\mathrm{v} / \mathrm{v})$, to achieve the content of exactly $5 \mathrm{mg}$ GAE per $1 \mathrm{~mL}$ sample. The new (40\%) ethanolic extract was sonicated 15 minutes at room temperature, then was filtered on the glass fiber filter. The resulting brown, homogeneous solution (Slae26) was used in pharmacological studies.

Polyphenol's assessment in extracts: Total phenols content was estimated using Folin Ciocalteau reagent, Romanian Pharmacopoeia (F.R.X) method [35]; results were expressed as $\mathrm{mg}$ gallic acid equivalents [GAE] per $1 \mathrm{~mL}$ sample $\left(\mathrm{R}^{2}=0.9912\right)$. Polyphenols profile in 70\% stokes aster ethanolic extract was assessed by Wagner and Bladt [36] and Reich and Schibli [37] thin layer chromatography recommendation; the general method for polyphenols assessment was used (solvent system: ethyl acetate-glacial acetic acid-formic acid-water/100:12:12:26), as described in the previous work [33].

Pharmacological studies in vitro: In vitro cell pharmacological studies were done on the human cancer colon cells Caco2 (ATCC-HTB-37), using MTS test [38]. Studies were designed to evaluate both, cytotoxic activity and antiproliferative potency of singular vegetal extract Slae26, of singular polyphenol compounds (reference substances, ref.), and of Slae26 combinations with each reference substance designated for the study. The combination of the test vegetal extract (Slae26) with the five reference substances was designed to provide 1:1 mass ratio $(\mathrm{w} / \mathrm{w})$ between the total phenols in the plant extract (expressed as $\mu \mathrm{g}$ GAE / $\mathrm{mL}$ sample) and the amount of the reference compound tested, the punctual concentrations in test series of $10,25,50$ and $100 \mu \mathrm{g}$ total active compounds per $1 \mathrm{~mL}$ sample respectively. Punctually, three test series and corresponding control samples were prepared: 1) Slae26 test series and Slae26 solvent sample (40\% ethanol) control series; 2) polyphenol compounds test series (ref.) and their solvent sample (70\% ethanol) control series; 3) Slae26 plus reference compound test series, 1:1 mass rate (w/w), and their solvent sample ( $40 \%$ ethanol plus $70 \%$ ethanol, $1: 1, \mathrm{w} / \mathrm{w}$ ) control series. Briefly, in the MTS cytotoxicity assay, Caco2 cells were exposed to the test/control sample series $(1,2,3)$ at the time when about approximately $70 \%$ "semi-confluent" cell culture had occurred, and in the antiproliferative MTS assay, Caco2 cells were exposed to three - test 
series at the time when about approximately 30\%" sub-confluent" cell culture had occurred. After one/ or two division cell cycles (24 and/or 48 hours) in the presence of test/control sample series, the culture medium is removed and Caco2 cells are further incubated with MTS solution for another 2 hours. At the end, the viability of the adherent cells is determined using CellTiter 96 AQueous One Solution Cell Proliferation Assay (Promega, USA) [38]. The absorbance of the treated and control series samples is therefore measured at $490 \mathrm{~nm}$ with a Microplate Reader (Chameleon V Plate Reader, LKB Instruments) and the recorded values were used for cell viability estimation in vitro (see formula below). Results were presented as punctual $\mathrm{DO}_{490} \mathrm{~nm}($ mean $\pm \mathrm{SD}, \mathrm{n}=3)$, and not as viability percent, to better emphasizes the influence of ethanol solvent in samples.

Molecular docking study: Molecular docking simulations were conducted using CLC Drug Discovery Workbench (QIAGEN) in order to evaluate the main phenolic derivatives Slae26 (luteolin-7-O-glcoside, luteolin-8-C-glcoside, caffeic acid, gentisic acid and p-aminobenzoic acid) as potential tankyrase enzyme inhibitors. The catalytic domain of the tankyrases (TNKS) is involved in oncogenesis, through Wnt signaling pathway responsible for regeneration of intestinal epithelial cells [39]. Testing new molecules with satisfactory physical-chemical properties by in silico methods against human tankyrases to block the Wnt pathway, constitutes a reliable approach to hit-to-lead stage of rational drug development for intestinal cancer treatment. Starting from this premise, the PDB entree $4 \mathrm{~W} 6 \mathrm{E}$, corresponding to human Takyrase 1 in complex to a small molecule inhibitor (encoded 3J5) [40], was retrieved form Protein Data Bank (https://www.rcsb.org). The protein preparation was realized by: removing the co-factor ( $\mathrm{Zn}$ ion) and the water molecules; protonation, setup binding site and binding pocket at $140.29 \AA^{2}$. Ligand's preparation was achieved by energy minimization using Spartan'14 software program from Wavefunction, Inc., Irvine, CA, U.S.A [41]. Validation was made by re-docking the native ligand 3J5. Interactions within the complex TNKS1-3J5 were identified.

\section{Results}

\subsection{Chemical analytical aspects of ethanolic extracts from Stokesia laevis}

Slae26 is (40\%) ethanolic extract standardized in total phenols content (5 mg GAE / mL) originated from the first (70\%) ethanol extract (Slae) from the aerial part of Stokesia laevis; the first extraction in $70 \%$ ethanol is done for reasons of technological sterilization and maximum extraction of polyphenols in plant material and the second extraction (solubilization) in $40 \%$ ethanol is done for reasons of punctual extract standardization.

\subsubsection{Polyphenol's appraisal in extracts}

- Figure 1 (chromatogram a and chromatogram b) presents polyphenols profile of Slae and Slae26 ethanolic extracts. Therefore, HPTLC analyses of the two ethanolic extracts from Stokesia laevis plant material (Slae/a and Slae26/b) indicated identical qualitative aspects, and the prevalence of two main polyphenols subclasses: caffeic acid derivates (blue fluorescent/fl. spots s3 and s5) namely chlorogenic acid (s3) and isochlorogenic acid (s5), and several luteolin derivates (yellow fl. spots s1, s2, s4 and s6); the major compound in stokes aster ethanolic extracts is in the category of luteolin monoglycosides (punctually, luteolin 7-O-glucoside/s4). Also, HPTLC study suggests the presence of small quantities of ellagic acid in stokes aster, punctually the blue, fl. spot at the start point of chromatograms a and b, more evident in $(70 \%)$ unrefined ethanolic extract Slae, than in standardized (40\%) ethanolic extract Slae26.

\subsection{Pharmacological in vitro cytotoxicity and antiproliferative results}

In vitro pharmacological studies were done on human colon cancer cell line Caco2 (ATCC-HTB-37); the selection of the intestinal cells was claimed by the precondition of direct contact between the food-related active compounds (ref.) and the affected tissue. Tests were done on Slae26, on the five reference compounds selected for the studies 
(punctually, luteolin 7-O-glucoside, luteolin 8-C-glucoside, caffeic acid, gentisic acid and para-aminobenzoic acid/PABA), and their combinations in a manner that ensured a quantitative ratio 1:1 between the active compounds (GAE face to particular reference compound) at identical concentrations and dilution series (punctually, 10, 25, 50 and 100 $\mu \mathrm{g}$ active compounds / $1 \mathrm{~mL}$ sample); they were in parallel with a negative control sample (blank) and the solvent samples control series, $40 \%$ ethanol series for Slae26 series and $70 \%$ ethanol series for the reference compounds series, respectively. It must be reminded that the results were presented as punctual O.D.490nm (mean $\pm S D, n=3$ ), and not as current viability percent, to better emphasizes the influence of ethanol solvent in samples.

\subsubsection{In vitro cytotoxicity MTS results at 24 hours and 48 hours}

- Cytotoxicity tests on $\mathrm{Caco} 2$ cells have at the purpose to evaluate the effects of the five reference compounds (luteolin 7-O-glucoside, luteolin 8-C-glucoside, caffeic acid, gentisic acid and PABA) at punctual dilution series (10, 25, 50 and $100 \mu \mathrm{g} / 1$ $\mathrm{mL}$ sample) comparatively to the solvent sample control series (70\% ethanol series), and the negative control sample (blank) respectively. Thereby, the cytotoxicity tests also considered the evaluation of the effects of ethanol solvent in culture medium.

- The cytotoxicity results at $24 \mathrm{~h}$ (Figure 2 ) indicated that, in comparison with the negative control sample (blank), all reference compounds at concentrations smaller than $25 \mu \mathrm{g} / \mathrm{mL}$ induced a stimulatory effect upon the viability of Caco2 cells, after that they induced an inhibitory effect. The cytotoxicity results on Caco2 cell viability at $48 \mathrm{~h}$ (Figure 3) shown certain inhibitory effects for all reference compounds tested and at all point series, but considering the augmented inhibitory effects of the solvent sample control series ( $70 \%$ ethanol), the only conclusion to be drawn is the protective effect of luteolin-7-O-glucoside, caffeic acid and gentisic acid against the cytotoxic effects of ethanol in culture medium; in the concentration interval less than $50 \mu \mathrm{g} / 1 \mathrm{~mL}$ sample), caffeic acid and gentisic acid only were effective against the alcohol damages in the intestinal cells.

\subsubsection{In vitro antiproliferative MTS test results at 48 hours}

- Antiproliferative MTS tests were done on Slae 26 ethanolic extract, on the five polyphenols compounds (reference substances, ref.) and their combinations made to assure 1:1 quantitative ratio $(\mathrm{w} / \mathrm{w})$ between the active compounds in samples. Figure 4 presents antiproliferative activity of the five polyphenols compounds (ref.) on Caco2 cells in comparison with to the solvent sample control series (70\% ethanol), 48 hours after the treatment. Figure 5 presents antiproliferative activity of the test vegetal extract Slae26 on human colon cancer cell line Caco2 (present study), and human breast cancer cell line BT20 [33] and murine melanoma cell line B16 [34], in comparison with the solvent sample control series ( $40 \%$ ethanol), 48 hours after the treatment. Figure 6 presents antiproliferative activity of the combinations of Slae26 and the five polyphenols compounds on human colon cancer cell line $\mathrm{Caco} 2$, in comparison with the solvent sample control series (70\% ethanol and 40\% ethanol, 1:1, $\mathrm{w} / \mathrm{w}), 48$ hours after the treatment.

- Therefore, referring to the antiproliferative potency of the five reference compounds tested (Figure 4), conclusions can be drawn only in the range of 10-25 $\mu \mathrm{g} / 1 \mathrm{~mL}$ sample; after the threshold of $25 \mu \mathrm{g} / \mathrm{mL}$ sample the effects being sensible alike to those of the control 70\% ethanol sample series; at concentrations smaller than $25 \mu \mathrm{g} /$ $1 \mathrm{~mL}$ sample, gentisic acid, caffeic acid, para aminobenzoic acid and 7-O-luteolin glucoside indicated certain inhibitory effects upon $\mathrm{Caco} 2$ cells viability, therefore an antiproliferative activity against human tumor cancer cells Caco2.

- Slae26 test sample in comparison with $40 \%$ ethanol sample control series (Figure 4), also indicated certain inhibitory activity upon the viability of human tumor cancer cell Caco2 ( $\mathrm{IC}_{50}=36 \mu \mathrm{g}$ GAE / mL extract), therefore similar to that previously found 
for human tumor breast cell BT20 ( $\mathrm{IC}_{50}=42 \mu \mathrm{g}$ GAE / $\mathrm{mL}$ extract) [33] and murine melanoma cell line $\mathrm{B} 16$ ( $\mathrm{IC}_{50}=39 \mu \mathrm{g}$ GAE / mL extract) [34]. Also, results on reference compounds and Slae26 sustain the observation of active antiproliferative zone of plant compounds in the interval 10 - $50 \mu \mathrm{g}$ product / $1 \mathrm{~mL}$ sample.

- The antiproliferative MTS tests made on the combinations of Slae26 with the five reference compounds (Figure 6), also in comparison with the control ethanol sample series (represented by 1:1 mixture between $40 \%$ ethanol from Slae26 sample and $70 \%$ ethanol from reference compounds samples) indicated their massive inhibitory effects upon the viability of Caco2 cells and an $\mathrm{IC}_{50}$ value towards $5 \mu \mathrm{g}$ active compounds per $1 \mathrm{~mL}$ sample.

\subsection{In silico evaluation of reference compounds by docking simulations}

According to recent data [42], tankyrase 1 (TNKS1) and tankyrase 2 (TNKS2) are two homologous proteins involved in multiple pathological situations, diseases and cancer development in humans: the two proteins (enzymes) were found present in colon cancer, gastric cancer, bladder cancer, pancreatic cancer, and also breast and brain cancer. On the other hand, the human colorectal cancer is the second leading cause of cancer death worldwide [43], and statistic data have revealed that approximately $90 \%$ of worldwide human colorectal tumors show the hyper-activation of $\beta$-catenin signaling pathway, while the TNKS inhibitors were found through the regulation of the limiting factor of the $\beta$-catenin degradation [42]. More, in vitro and in vivo proof-of-concept studies established the profile of the selective inhibitors of TNKS in cancer and tankyrase linked diseases [44]. In this context, studying TNKS inhibitory potential of natural compounds is of great interest. Therefore, molecular docking simulation in the present work aimed to study potential inhibitory effects of the five phenolics in the series of food bioactive compounds, on the molecular target TNKS1, compared to the co-crystalized ligand coded Co-crystallized 3J5A, proved to be a potent inhibitor of TNKS enzymes.

- $\quad$ Results of molecular docking study (Table 1 and Table 2) on human tankyrase 1 (PDB ID: 4W6E) have revealed the greatest inhibitory score (-104.15) for the Co-crystallized 3J5A ligand: there were counted four hydrogen bond formation with GLU1291 (x2), SER1221 and GLY1185 amino acid residues (see Supplementary Figure S1). It is noticed that luteolin-7-O-glucoside strongly interacted with SER1221 and GLY1185 by hydrogen bonding and additionally with GLY1196 and ASP1198, achieving a docking score value of -80.49 , the best among the investigated ligands. Except Luteolin-8-C-glcoside, all molecules interact with GLY1185 and SER1221 (see Supplementary Figures S2-S6), meaning satisfactory docking scores.

\subsection{Figures}

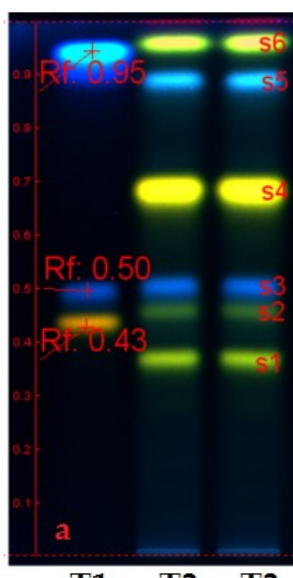

T1 12 T2

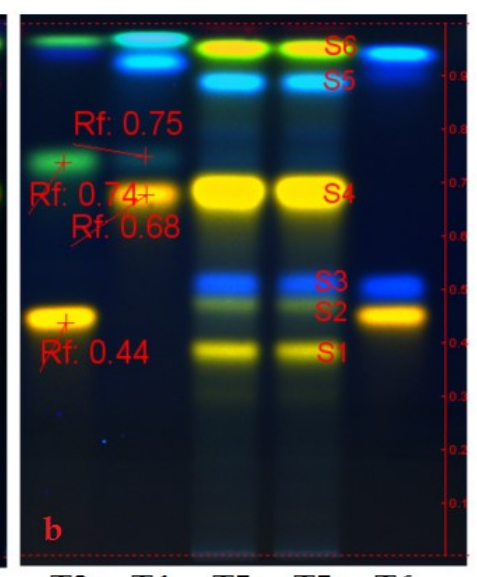

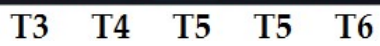

Figure 1 (a, b). Polyphenols profile in Stokesia laevis L. ethanolic extracts. 
Where: Chromatogram a. Track T1, rutin, chlorogenic acid and caffeic acid (ref.). Tracks T2 whole $(70 \%)$ ethanolic extract from Stokesia laevis (Slae) - duplicate sample; Chromatogram b. Track T3, rutin, vitexin, protocatechuic acid and apigenin (ref.); Track T4, hyperoside, cosmosiin, rosmarinic acid and kaempferol; Tracks T5 - standardized (40\%) ethanolic extract from Stokesia laevis (Slae26) - duplicate sample; Track T6, rutin, chlorogenic acid and caffeic acid (ref.).

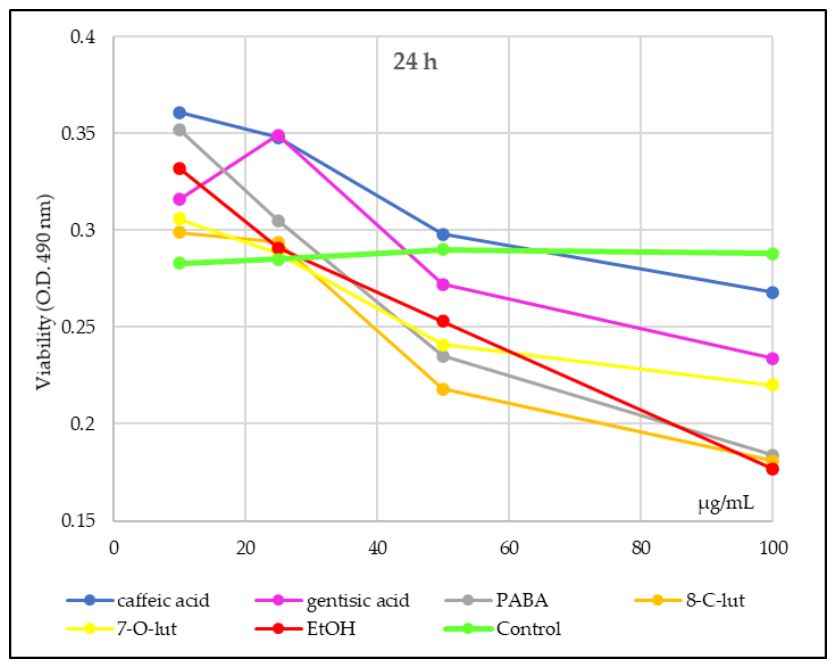

Figure 2. Caco2 cell viability treated with the five reference compounds (phenolics), at $24 \mathrm{~h}$

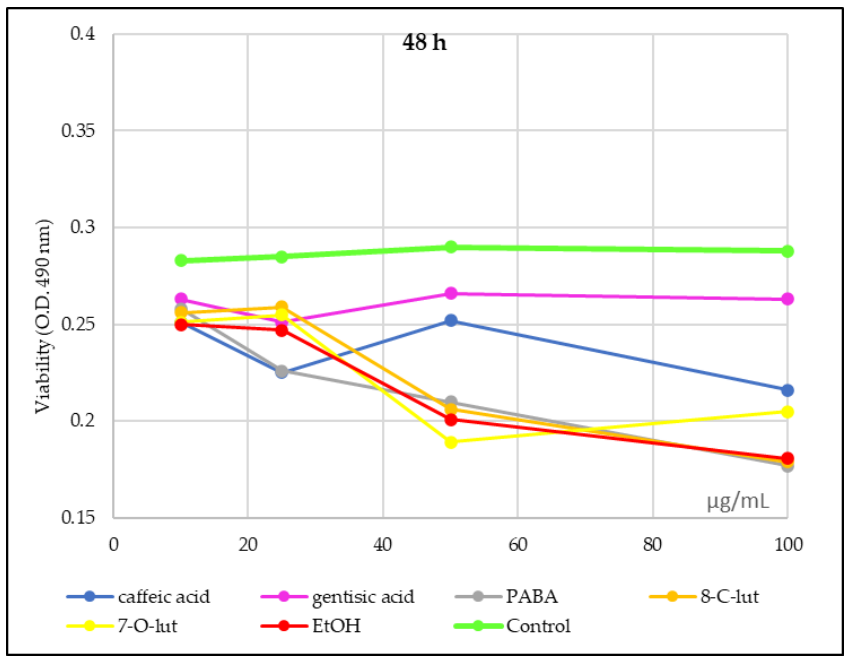

Figure 3. Caco2 cell viability treated with the five reference compounds (phenolics), at $48 \mathrm{~h}$ 


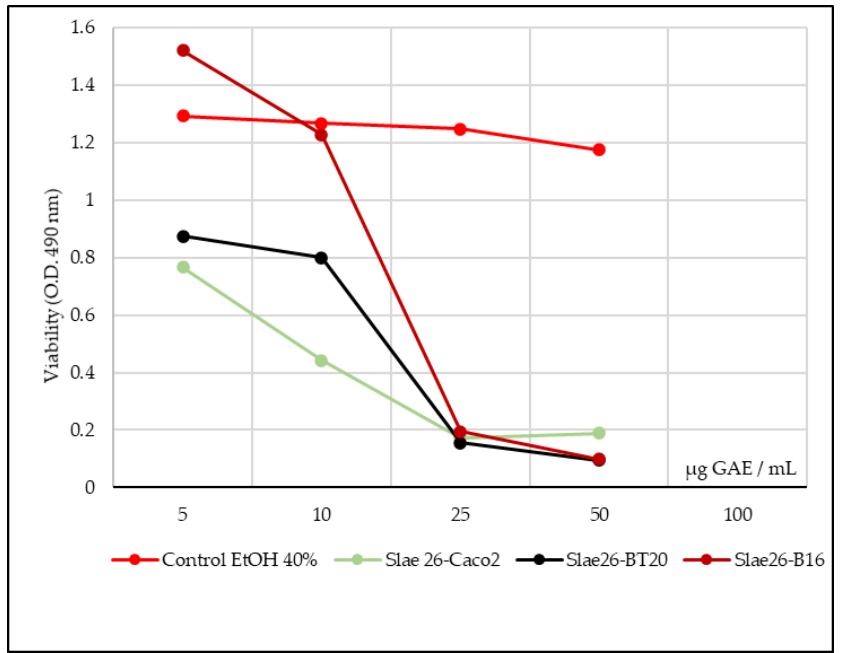

Figure 4. Caco2 antiproliferative activity of the five reference compounds (phenolics), at $48 \mathrm{~h}$

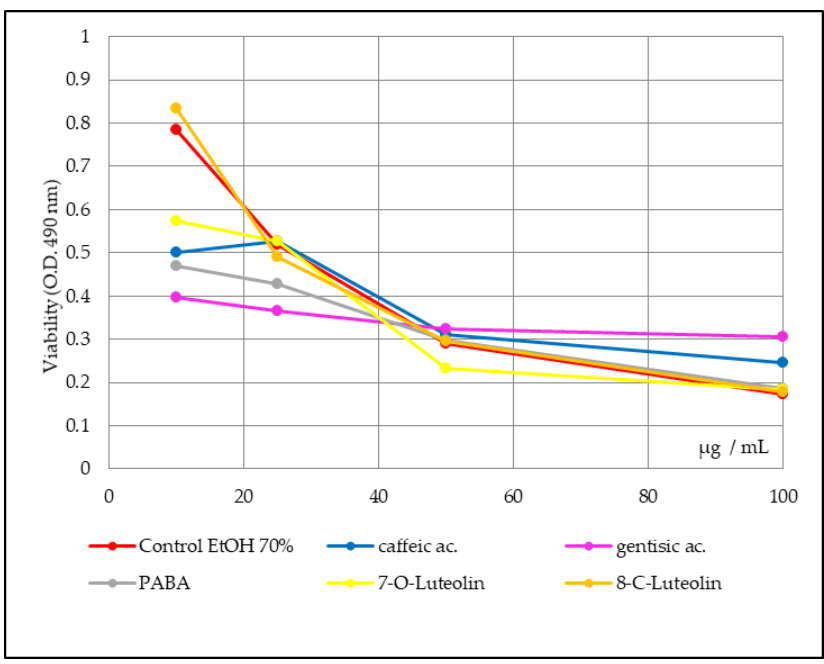

Figure 5. Antiproliferative activity of the Slae26, tested on Caco2, BT20 and B16 cell lines, at $48 \mathrm{~h}$

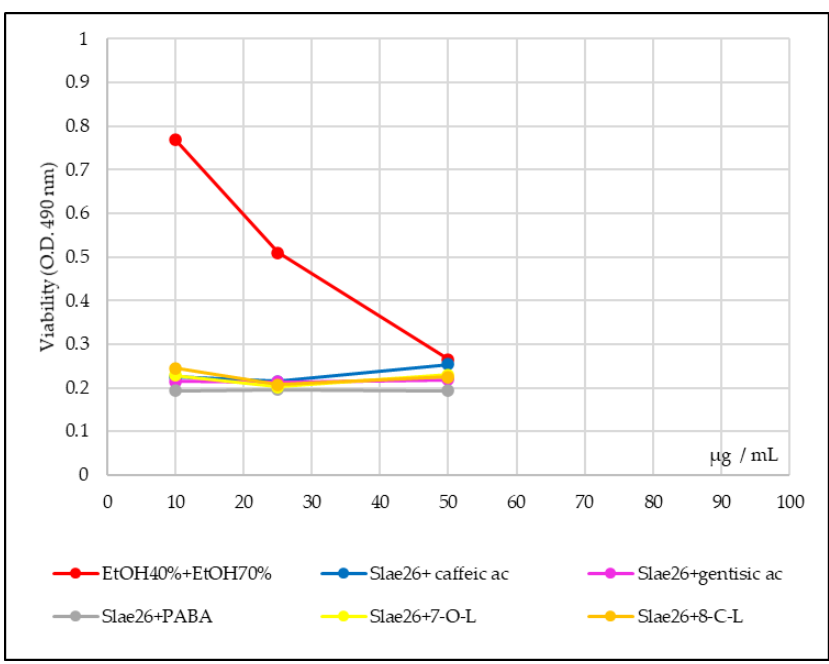

Figure 6. Caco2 antiproliferative activity of the five combinations of Slae26 with the five reference compounds tested (phenolics, 1:1 w/w ratio of the active compounds in series), at $48 \mathrm{~h}$ 
Table 1. Results of molecular docking study on human tankyrase 1 (PDB ID: 4W6E).

\begin{tabular}{|c|c|c|c|c|}
\hline Ligand & Score & RMSD & Hydrogen bond & Length $(\AA)$ \\
\hline \multirow[t]{4}{*}{ Co-crystallized 3J5A } & -104.15 & 0.16 & O1 sp³ - Osp² GLU1291 & 3.393 \\
\hline & & & O1 sp ${ }^{3}-$ Osp $^{2}$ GLU1291 & 3.250 \\
\hline & & & O sp $p^{2}-$ Osp $^{3}$ SER1221 & 2.778 \\
\hline & & & $\mathrm{O} \mathrm{sp}^{2}-\mathbf{N s p}^{2} \mathrm{GLY} \mathbf{1 1 8 5}$ & 2.895 \\
\hline \multirow[t]{10}{*}{ Luteolin-7-O-glcoside } & -80.49 & 0.69 & O10 sp ${ }^{3}-$ Osp $^{2}$ GLY1196 & 3.284 \\
\hline & & & O10 sp ${ }^{3}-\mathrm{Nsp}^{2}$ ASP1198 & 2.729 \\
\hline & & & O11 sp ${ }^{3}-$ Nsp$^{2}$ ASP1198 & 2.741 \\
\hline & & & O11 sp $\mathrm{sp}^{3}-\mathrm{Osp}^{2} \mathrm{ASP} 1198$ & 3.067 \\
\hline & & & O5 sp ${ }^{3}-$ Osp $^{2}$ GLY1185 & 2.456 \\
\hline & & & O3 sp ${ }^{3}-$ Osp $^{2}$ GLY1185 & 3.090 \\
\hline & & & O3 sp ${ }^{3}-$ Nsp $^{2}$ GLY1185 & 2.647 \\
\hline & & & O3 $\mathrm{sp}^{3}-$ Osp $^{3}$ SER1221 & 3.040 \\
\hline & & & O4 $\mathrm{sp}^{3}-$ Osp $^{3}$ SER1221 & 3.087 \\
\hline & & & O4 sp ${ }^{3}-\mathrm{Nsp}^{2}$ SER1221 & 3.298 \\
\hline \multirow[t]{5}{*}{ Luteolin-8-C-glcoside } & -61.88 & 0.02 & O11 sp ${ }^{3}-\mathrm{Nsp}^{2}$ ASP1198 & 2.992 \\
\hline & & & O9 $\mathrm{sp}^{2}-\mathrm{Nsp} \mathrm{p}^{2} \mathrm{TYR} 1213$ & 3.034 \\
\hline & & & O4 sp ${ }^{3}-\mathrm{Nsp}^{2}$ HIS1201 & 2.920 \\
\hline & & & O3 sp ${ }^{3}-$ Osp $^{2}$ ALA1202 & 3.129 \\
\hline & & & O6 sp ${ }^{3}-$ Nsp $^{2}$ HIS1201 & 2.817 \\
\hline \multirow[t]{6}{*}{ Caffeic acid } & -58.55 & 0.15 & O3 sp ${ }^{3}-$ Osp $p^{3}$ TYR1224 & 3.050 \\
\hline & & & O3 sp ${ }^{3}-$ Osp $^{2}$ GLU1291 & 2.912 \\
\hline & & & O2 sp ${ }^{3}-$ Osp 2 TYR1213 & 3.364 \\
\hline & & & O2 sp ${ }^{3}-\mathrm{Nsp}^{2} \mathrm{HIS1184}$ & 3.089 \\
\hline & & & $\mathrm{O} 2 \mathrm{sp}^{3}-\mathrm{Nsp}^{2}$ GLY1185 & 2.925 \\
\hline & & & $\mathrm{O} 2 \mathrm{sp}^{3}-\mathrm{Osp}^{2} \mathrm{GLY1185}$ & 3.022 \\
\hline
\end{tabular}




\begin{tabular}{|c|c|c|c|c|}
\hline \multirow[t]{6}{*}{ Gentisic acid } & -51.02 & 0.01 & O1 sp ${ }^{3}-$ Osp ${ }^{3}$ TYR1224 & 3.142 \\
\hline & & & O0 sp ${ }^{3}-$ Osp $p^{2}$ TYR1213 & 3.212 \\
\hline & & & O0 sp $\mathrm{s}^{3}-\mathrm{Osp}^{3}$ SER1221 & 2.880 \\
\hline & & & O2 $\mathrm{sp}^{3}-\mathrm{Nsp}^{2} \mathrm{HIS} 1184$ & 3.196 \\
\hline & & & O2 sp ${ }^{3}-$ Nsp $^{2}$ GLY1185 & 3.123 \\
\hline & & & $\mathrm{O} 2 \mathrm{sp}^{3}-\mathrm{Osp}^{2}$ GLY1185 & 2.829 \\
\hline \multirow[t]{7}{*}{ p-aminobenzoic acid } & -49.16 & 0.17 & N2 $\mathrm{sp}^{3}-$ Osp ${ }^{3}$ TYR1224 & 3.092 \\
\hline & & & O0 sp ${ }^{3}-$ Osp $p^{2}$ TYR1213 & 3.087 \\
\hline & & & $\mathrm{O} 0 \mathrm{sp}^{3}-\mathrm{Nsp}^{2}$ ALA1225 & 3.270 \\
\hline & & & O0 $\mathrm{sp}^{3}-\mathrm{Osp}^{3}$ SER1221 & 2.678 \\
\hline & & & O0 sp ${ }^{3}-$ Osp $^{2}$ PHE1183 & 2.989 \\
\hline & & & O1 sp $\mathrm{sp}^{2}-\mathrm{Nsp}^{2} \mathrm{HIS1184}$ & 3.037 \\
\hline & & & O1 sp ${ }^{2}-$ Nsp $^{2}$ GLY1185 & 3.001 \\
\hline
\end{tabular}

Table 2. Lipinski's parameters for druggability assessment.

\begin{tabular}{cccccc}
\hline Ligand & HBD & HBA & LogP & LV & rb \\
\hline Co-crystallized 3J5A & 1 & 7 & 4.66 & 0 & 4 \\
Luteolin-7-O-glcoside & 7 & 11 & 1.57 & 2 & 4 \\
Luteolin-8-C-glcoside & 8 & 11 & -0.70 & 2 & 3 \\
Caffeic acid & 3 & 4 & 1.58 & 0 & 2 \\
Gentisic acid & 3 & 4 & 1.49 & 0 & 1 \\
p-aminobenzoic acid & 3 & 3 & 0.97 & 0 & 1 \\
\hline
\end{tabular}

" HBD - hydrogen bond donors; HBA- hydrogen bond acceptors; logP- water-octanol partition coefficient ; LV-Lipinski's violations; rb-rotatable bonds count .

\section{Discussion}

The objectives proposed in the present work were to evaluate the antiproliferative activity on human colon cancer cell $\mathrm{Caco} 2$ of the ethanolic extract from Stokesia laevis plant species (codified Slae26), of five food-related polyphenols compounds (reference substances, ref.) selected based on precise criteria, and of Slae 26 combinations with these in a manner that ensured 1:1 mass rate $(\mathrm{w} / \mathrm{w})$ between the active compounds in the combined samples. The selection of the five polyphenols compounds, namely luteolin-8-C-glucoside (orientin), luteolin-7-O-glucoside (cinnaroside), caffeic acid, gentisic acid and para-amino benzoic acid (PABA) is supported by the following criteria: Luteolin monoglycosides are the major flavonoid compounds in Stokesia laevis ethanolic extracts, and the main contributor on antiproliferative activity of Slae26 in the authors' 
works [33-34], as results from the literature data today [45-49]; Caffeic acid is the foremost compound in human diet resulting from the metabolism of (iso)chlorogenic acid esters, also the subject of active transport in humans, therefore it can be considered the food-related bioactive compound with the largest and constant presence in the human intestine; moreover, studies proved its ability to protect the human peripheral blood lymphocytes against the cellular damages induced by gamma radiation (gamma radiation is used in radio-oncology to treat cancer patients), therefore the combination of a natural antiproliferative product with a natural radiation guardian is of interest for humans [50]; Gentisic acid is a highly active antioxidant compound proved to enhance the activity of antioxidant enzymes in humans at the same time acting as an inhibitor of lipid peroxidation in cell membranes, also demonstrated to effectively protect human erythrocytes against gamma radiation exposure [51-53]; Benzoic acid is a natural phenolic acid with antibacterial activity [54] used as a preservative in food [55]; it also can be found in fermentation products such as Caciocavallo and mozzarella cheeses [56].

In this context, cytotoxicity MTS tests were designed to investigate both, the effects of the five reference compounds applied on Caco2 cells at the time when about $70 \%$ of the predicted "sub-confluent" cells had occurred, and the effects on Caco2 cells viability of ethanol solvent in the test samples. Strong inhibitory effects of control $(70 \%)$ ethanol solvent series on the viability of $\mathrm{Caco} 2$ cells, more augmented than that of the test samples, made it impossible to draw a conclusion on the cytotoxicity of the reference compounds; yet, caffeic acid and gentisic acid graph aspect at $24 \mathrm{~h}$ and $48 \mathrm{~h}$, both suggest their ability to protect intestinal cells against the cytotoxic effects of ethanol solvent.

Antiproliferative MTS tests on the five reference compounds, applied on Caco2 cells at the time when about $30 \%$ of the predicted "sub-confluent" cells had occurred, indicated certain antiproliferative effects of gentisic acid, caffeic acid, para-aminobenzoic acid (PABA) and 7-O-luteolin glucoside in the range of $10-25 \mu \mathrm{g} / \mathrm{mL}$ sample, after that the activity of the reference compounds series being substantially matching with that of the control solvent $(70 \%$ ethanol) dilution series. Slae26 test sample in comparison with own control solvent ( $40 \%$ ethanol) dilution series indicated certain inhibitory activity of the vegetal extract upon the viability of the human tumor cancer cell Caco2 $\left(\mathrm{IC}_{50}=36 \mu \mathrm{g}\right.$ GAE / $\mathrm{mL}$ sample.), therefore similar to that previously found for human breast cancer cell line BT20 (IC $50=42 \mu \mathrm{g}$ GAE / mL sample) [33] and murine melanoma cell line B16 (IC $50=39 \mu \mathrm{g}$ GAE / $\mathrm{mL}$ sample) [34]. Slae26 combinations with the five reference compounds (1:1 ratio between the active compounds in samples) in comparison with the control ethanol sample series (1:1 ratio between 40\% ethanol solvent in Slae26 sample and 70\% ethanol solvent in reference samples) indicated significant increases of inhibitory effects upon the viability of Caco2 cells (IC50 $5 \mu \mathrm{g} / \mathrm{mL}$ sample), without interfering toxic effects of the ethanol solvent in samples. Punctually, the IC50 value of Skae26 was reduced from $36 \mu \mathrm{g} /$ $\mathrm{mL}$ sample to about $5 \mu \mathrm{g} / \mathrm{mL}$ sample, signifying real therapeutic usefulness and antitumor potency on human colon cancer cell of Sla26 combinations with the five food-related bioactive compounds.

Furthermore, docking studies on the molecular target TNKS1 indicated the greatest inhibitory activity (score value: -104.15 ) for the Co-crystallized 3J5A and interactions (hydrogen bonds) with three amino acid residues in the active binding site (SER1221, GLY1185, and GLY1196). Luteolin-7-O-glucoside also strongly interacted with the TNKS1 target, punctually established interactions with two of three amino acids above (SER1221, GLY1185), achieving a docking score value of -80.49. The other four food bioactive compounds indicated score values of -68.88 for luteolin-8-C-glcoside, -58.55 for caffeic acid, -51.02 for gentisic acid and -49.16 for para-aminobenzoic acid. Overall, docking studies on the human tankyrase 1 (TNKS1) confirmed antiproliferative potency of bioactive compounds from vegetal sources and food products. 
On the other hand, studying the transport of caffeic and chlorogenic acids in Caco2 cells, Kobayashi et al [57] noticed a greater efficiency of absorption of caffeic acid, and the fact that polarized transport of caffeic acid has been inhibited by benzoic acid and acetic acid, two representative substrates of MCT (monocarboxylic acid transporter) in the intestine. They also revealed that the intestinal transport took place mainly by paracellular diffusion, and that $\mathrm{m}$-coumaric acid and 3-(m-hydroxyphenyl) propionic acid, the main metabolites of chlorogenic and caffeic acid through the colonic microflora, competitively inhibited the transport of fluorescein, also known as a substrate of MCT. Together, these results proved the physiological importance of MCT-mediated absorption, both in the case of phenolic acids themselves and their colonic metabolites. Furthermore, aiming to investigate antioxidant activity of combination of luteolin and chlorogenic acid, Hsieh et al studies [58] indicated that the half maximal inhibitory concentration of luteolin and chlorogenic (IC 50 of luteolin was estimated at about $26 \mu \mathrm{g} \cdot / \mathrm{mL}$ and of chlorogenic acid at about $86 \mu \mathrm{g} / \mathrm{mL}$ ) combined in a 1:1 ratio resulted in an augmented increase of free radical oxygen inhibition (78\%), compared to that of separate compounds. Finally, in vivo studies [59] on the diabetic rats with gastroentheropaty induced by NSAIDS (Diclofenac sodium/DCF; $7.5 \mathrm{mg} / \mathrm{kg}$, PO, b.i.d.) indicated that kaempferol, ascorbic acid, lupeol, diosgenin, $\beta$-sitosterol, stigmasterol and $\beta$-amyrin acted synergistically in combination with Costus pictus ethanolic extract $(\mathrm{CP})$; the punctual combination of quercetin $(\mathrm{Q})$ with Costus pictus ethanolic extract (QCT; $50 \mathrm{mg} / \mathrm{kg}$ for total 10 days) indicating it would be a promising candidate for treating patient with type 2 diabetes mellitus and NSAID induced - gastroenteropathy.

\section{Conclusions}

Further studies to assess the evolution of antiproliferative activity of Slae26 combinations with polyphenols compounds upon the tumor cell lines Caco2, BT20 and B16, at lower concentrations and on several consecutive days, are proposed.

Supplementary Materials: The following are available online at www.mdpi.com/xxx/s1, Figure S1: Hydrogen bond interactions of Co-crystallized 3J5A with TNKS1, Figure S2: Hydrogen bond interactions of luteolin-7-O-glcoside with TNKS1, Figure S3: Hydrogen bond interactions of luteolin-8-C-glcoside with TNKS1, Figure S4: Hydrogen bond interactions of caffeic acid with TNKS1, Figure S5: Hydrogen bond interactions of gentisic acid with TNKS1, Figure S6: Hydrogen bond interactions of p-aminobenzoic acid with TNKS1.

Author Contributions: conceptualization, L.C.P.; methodology, G.N. and A.A.; formal analysis, A.S. and L.P.; investigation. G.N., A.A. and L.C.P.; resources, L.C.P.; writing-original draft preparation, L.C.P.; writing - review and editing, L.C.P. and A.S.; visualization, A.S.; supervision, L.C.P.; project administration, L.C.P.; funding acquisition, L.C.P.

Funding: This work was supported by the ANCSI program POC-A1-A1.2.3-G-2015, Project title "New technologies and natural derived products for human health use", Contract no.60/05.09.2016, ID P_40_406, SMIS 105542.

Conflicts of Interest: The authors declare no conflict of interest.

\section{References}

1. Harborne, J.B. Classes and functions of secondary products from plants. Chemicals from Plants, Perspectives on Secondary Plant Products. Walton, N.J., Brown D.E., Eds.; Imperial College Press: London, UK: 1999. pp. 1-25.

2. Pott, D.M.; Osorio, S.; Vallarino, J.G. From Central to Specialized Metabolism: An Overview of Some Secondary Compounds Derived From the Primary Metabolism for Their Role in Conferring Nutritional and Organoleptic Characteristics to Fruit. Front Plant Sci. 2019, 10, Article 835, 1-19. 
3. Carrington, Y.; Guo J.; Fillo, A.; Kwon, J.; Tran, L.T.; Ehlting, J.; Fernie, A.R; Nikoloski, Z.; Osorio, S. Evolution of a secondary metabolic pathway from primary metabolism: shikimate and quinate biosynthesis in plants. Plant J. 2018, 95, 823-833.

4. Weng, J.K. The evolutionary paths towards complexity: a metabolic perspective. New Phytol. 2013, 201, 1141-1149.

5. Vallarino, J.G.; de Abreu e Lima, F.; Soria, C.; Tong, H.; Pott, D.M.; Willmitzer, L. et al. (2018). Genetic diversity of strawberry germplasm using metabolomic biomarkers. Sci. Rep. 2018, 8:14386.

6. Wink, M. Evolution of secondary metabolites from an ecological and molecular phylogenetic perspective. Phytochemistry. 2003, 64, 3-19.

7. Bennett, R.N.; Wallsgrove, R.M. Secondary metabolites in plant defense mechanisms. New Phytol. 1994, 127, $617-633$.

8. Kroymann, J. Natural diversity and adaptation in plant secondary metabolism. Current Opin Plant Biol. 2011, 14, 246-51.

9. Yang, L.; Wen, K.S.; Ruan, X.; Zhao, Y.X.; Wei, F.; Wang, Q. Response of Plant Secondary Metabolites to Environmental Factors. Molecules, 2018, 23, 762.

10. Cragg, G.M.; Newman, D.J.; Snader, K.M. Natural products in drug discovery and development. J. Nat. Prod. 1997, 60, 52-60.

11. Cragg, G.M.; Newman, D.J. Natural Products as Sources of New Drugs over the Nearly Four Decades from 01/1981 to 09/2019. J. Nat. Prod. 2020, 83, 770-803.

12. Newman, D.J.; Cragg G.M. Natural Products as Sources of New Drugs over the 30 Years from 1981 to 2010. J. Nat. Prod. 2012, 75, 311-335.

13. Grabley, S.; Thiericke, R. Bioactive agents from natural sources: trends in discovery and application. Adv Biochem Eng Biotechnol. 1999, 64, 101-54.

14. Lipinski, C.A. Lead- and drug-like compounds: the rule-of-five revolution. Drug Discov Today. Technol. 2004, 1, 337-341.

15. Macarron, R. Critical review of the role of HTS in drug discovery. Drug Discov Today. 2006, 11, $277-279$.

16. Keller, T.H.; Pichota, A.; Yin, Z. A practical view of 'druggability. Curr Opin Chem Biol. 2006, 10, 357-361.

17. Perez-Jimenez, J.; Neveu, V.; Scalbert, A. Identification of the 100 richest dietary sources of polyphenols: an application of the Phenol-Explorer database. Eur J Clin Nutr. 2010, 64 Suppl 3: S112-20.

18. Neveu, V.; Perez-Jimenez, J.; Vos, F.; Crespy, V.; Chaffaut, L.; Mennen, L.; Knox, C.; Eisner, R.; Cruz, J.; Wishart, D.; Scalbert, A. Phenol-Explorer: an online comprehensive database on polyphenol contents in foods. Database (Oxford) 2010, 2010: bap024.

19. Manach, C.; Scalbert, A.; Morand, C.; Rémésy, C.; Jiménez, L. Polyphenols: food sources and bioavailability. Am J Clin Nutr. 2004, 79, 727-747.

20. Manach, C.; Williamson, G.; Morand, C.; Scalbert, A.; Remesy, C. Bioavailability and bioefficacy of polyphenols in humans. I. Review of 97 bioavailability studies. Am J Clin Nutr 2005, 81, 230S-42S.

21. Rein, M.J.; Renouf, M.; Cruz-Hernandez, C.; Actis-Goretta, L.; Thakkar, S.K.; Silva Pinto, M. Bioavailability of bioactive food compounds: a challenging journey to bioefficacy. Br J Clin Pharmacol 2013, 75, 588-602.

22. Scalbert, A.; Williamson, G. Dietary intake, and bioavailability of polyphenols. J Nutr. 2000, 130, 2073S-2085.

23. Konishi, Y.; Kobayashi, S. Microbial metabolites of ingested caffeic acid are absorbed by the monocarboxylic acid transporter (MCT) in intestinal Caco-2 cell monolayers. J Agric Food Chem 2004, 52, 6418-24.

24. Saura-Calixto, F.; Serrano, J.; Goñi, I. Intake and bioaccessibility of total polyphenols in a whole diet. Food Chem. 2007, 101, 492-501. 
25. Neilson, A.P.; Ferruzzi, M.G. Influence of formulation and processing on absorption and metabolism of flavan-3-ols from tea and cocoa. Annu Rev Food Sci Technol. 2011, 2, 125-151.

26. Atanasov, A.G.; Zotchev, S.B.; Dirsch, V.M.; Supuran, C.T. Natural products in drug discovery: advances and opportunities. Nat. Rev. Drug Discov. 2021, 20, 200-216.

27. Li, J.W.H.; Vederas, J.C. Drug discovery and natural products: end of an era or an endless frontier? Science 2009, 325, 161-165.

28. Clardy, J.; Walsh, C. Lessons from natural molecules. Nature 2004, 432, 829-837.

29. Kuppusamy, P.; Yusoff, M.M.; Parine, N.R.; Govindan, N. Evaluation of in-vitro antioxidant and antibacterial properties of Commelina nudiflora L. extracts prepared by different polar solvents. Saudi J Biol Sci 2015, 22, $293-301$.

30. Akkari, H.; Hajaji, S.; B'chir, F.; Rekik. M.; Gharbi, M. Correlation of polyphenolic content with radical-scavenging capacity and anthelmintic effects of Rubus ulmifolius (Rosaceae) against Haemonchus contortus. Vet Parasitol 2016, 221, 46-53.

31. Bartmanska, A.; Walecka-Zacharska, E.; Tronina, T.; Poplonski, J.; Sordon, S.; Brzezowska, E.; Bania, J.; Huszcza, E. Antimicrobial Properties of Spent Hops Extracts, Flavonoids Isolated Therefrom, and Their Derivatives. Molecules 2018, 23, 2059.

32. Rasoanaivo, P.; Wright.; C.V.; Willcox, M.L.; Gilbert, B. Whole plant extracts versus single compounds for the treatment of malaria: synergy and positive interactions. Malaria Journal 2011, 10(Suppl 1): S4.

33. Pirvu, L.; Neagu, G.; Terchescu, I.; Albu, B.; Stefaniu, A. Comparative studies on two vegetal extracts from Stokesia laevis and Geranium pratense; polyphenols profile, cytotoxic effect and antiproliferative activity. Open Chem. 2020, $18,488-502$.

34. Neagu, G.; Stefaniu, A.; Albu, B.; Terchescu, I.; Pintilie, L.; Pirvu, L. Stokesia aster ethanolic extract activity on the normal and malignant murine cell line viability L969 and B16. Chem. Proc. 2021, 3, 42.

35. Collective work. Romanian Pharmacopoeia, Xth ed., Medicala, Bucharest, 1993.

36. Wagner, H.; Bladt, S. Plant Drug Analysis. A Thin Layer Chromatography Atlas, 2nd ed.; Springer-Verlag: Berlin Heidelberg, 1996.

37. Reikh, E.; Schibli, A. HPTLC for the Analysis of Medicinal Plants. Thieme, N.Y.-Stuttgart, 2008.

38. https://www.promega.ro.pdf

39. Pinto, D.; Gregorieff, A.; Begthel, H.; Clevers, H. Canonical Wnt signals are essential for homeostasis of the intestinal epithelium. Genes Dev. 2003, 17, 1709-13.

40. Johannes, J.W.; L. Almeida, L.; Barlaam, B.; Boriack-Sjodin, P. A.; Casella, R.; Croft, R. A.; Dishington, A. P.; Gingipalli, L.; Gu, C.; Hawkins, J. L.; Holmes, J. L.; Howard, T.; Huang, J.; Ioannidis, S.; Kazmirski, S.; Lamb, M.L.; McGuire, T. M.; Moore, J.E.; Ogg, D.; Patel, A.; Pike, K. G.; Pontz, T.; Robb, G.R.; Su, N.; Wang, H.; Wu, X.; Zhang, H.-J. ; Zhang, Y.; Zheng, X.; Wang, T. Pyrimidinone Nicotinamide Mimetics as Selective Tankyrase and Wnt Pathway Inhibitors Suitable for in Vivo Pharmacology, ACS Med. Chem. Lett. 2015, 6, 254-259, Deposited on: 2014-08-20.

41. Shao, Y.; Molnar, L.F.; Jung, Y.; et al., Advances in methods and algorithms in a modern quantum chemistry program package, Phys. Chem. Chem. Phys. 2006, 8(27), 3172-3191.

42. Haikarainen, T.; Krauss, S.; Lehtio, L. Tankyrases: Structure, Function and Therapeutic Implications in Cancer. Curr Pharm Des. 2014, 20, 6472-6488.

43. Song, M.; Emilsson, L.; Bozorg, S.R.; Nguyen, L.H.; Joshi, A.D.; Staller, K; Nayor, J.; Chan, A.T.; Ludvigsson, J.F. Risk of colorectal cancer incidence and mortality after polypectomy: a Swedish record-linkage study. Lancet Gastroenterol Hepatol. 2020, 5, 537-47. 
44. Zamudio-Martinez, E.; Herrera-Campos, A.B.; Munoz, A.; Rodriguez-Vargas, J.M.; Olivier, F.V. Tankyrases as modulators of pro-tumoral functions: molecular insights and therapeutic opportunities. J Exp Clin Cancer Res 2021, 40, Article number: 144.

45. Baskar, A.A.; Ignacimuthu, S.; Michel, G.O.; Al Numair, K.S. Cancer chemopreventive potential of luteolin-7-O-glucoside isolated from Ophiorrhiza mungos Linn. Nutr Cancer 2011, 63, 130-8.

46. Goodarzi, S., Tabatebaei, M.J.; Jafari, R.M.; Shemirani, F.; Tavakoli, S.; Mofasseri, M.; Tofighi Z. Cuminum cyminum fruits as source of luteolin- 7-O-glucoside, potent cytotoxic flavonoid against breast cancer cell lines. Nat Prod Res 2020, 34, 1602-1606.

47. Palombo, R.; Caporali, S.; Falconi, M.; Iacovelli, F.; Della Roca, B.M.; Lo Surdo, A.; Campione, E.; Candi, E.; Melino, G.; Bernardini, S.; Terrinoni, A. Luteolin-7-O- $\beta$-D-Glucoside Inhibits Cellular Energy Production Interacting with HEK2 in Keratinocytes. Int. J. Mol. Sci. 2019, 20, 2689.

48. Park, S.H.; Kim, J.H.; Lee, D.H.; Kang, J.W.; Song, H.H.; Oh, S.R.; Yoon D.Y.; Luteolin 8-C- $\beta$-fucopyranoside inhibits invasion and suppresses TPA-induced MMP-9 and IL-8 via ERK/AP-1 and ERK/NF- $\kappa B$ signaling in MCF-7 breast cancer cells. Biochimie. 2013, 95, 2082-2090.

49. Lee, J.; Park, S.H.; Lee, J.; Chun, H.; Choi, M.K.; Yoon, J.H.; Pham, T.H.; Kim, K.H.; Kwon T.; Ryu, H.W.; Oh, S.R.; Yoon, D.Y. Differential effects of luteolin and its glycosides on invasion and apoptosis in MDA-MB-231 triple-negative breast cancer cells. EXCLI J. 2019, 18, 750-763.

50. Devipriva, N.; Sudheer, A.R.; Menon, V.P. Caffeic acid protects human peripheral blood lymphocytes against gamma radiation-induced cellular damage. J Biochem Mol Toxicol 2008, 22, 175-86.

51. Sana, N.; Sheikh, T.A.; Wani, A.W.; Summya, R.; Nemat, A..; Sarwat, S. Modulatory effects of gentisic acid against genotoxicity and hepatotoxicity induced by cyclophosphamide in Swiss albino mice. J Pharm Pharmacol. 2012, 64, 259:67.

52. Joshi, R.; Gangabhagirathi, R.; Venu, S.; Adhikari, S.; Mukherjee, T. Antioxidant activity and free radical scavenging reactions of gentisic acid: in-vitro and pulse radiolysis studies. Free Radic Res 2012, 46, 11-20.

53. Abedi, F.; Razavi, B.M.; Hosseinzadeh, H. A review on gentisic acid as a plant derived phenolic acid and metabolite of aspirin: Comprehensive pharmacology, toxicology, and some pharmaceutical aspects. Review. Phytotherapy Research 2019, 34, 729-741.

54. Daeschel, M.S. Antimicrobial substance in lactic acid bacteria for use as food preservatives. Food Technol. 1997, 1, 164-167.

55. https://www.sciencedirect.com/topics/agricultural-and-biological-sciences/benzoic-acid

56. Park, S.Y.; Yoo, M.Y.; Paik, H.D.; Lim, S.D. Production of benzoic acid as a natural compound in fermented skim milk using commercial cheese starter. J. Dairy Sci. 2017, 100, 4269-4275.

57. Kobayashi, S.; Konoshi, Y. Transepithelial transport of chlorogenic acid, caffeic acid, and their colonic metabolites in intestinal caco-2 cell monolayers. J Agric Food Chem. 2004; 52, 2518-26.

58. Hsieh, M.H.; Hsieh, M.J.; Wu, C.R.; Peng, W.H.; Hsieh, M.T.; Hsieh, C.C. The Synergistic Effect of Antioxidant Interaction between Luteolin and chlorogenic Acid in Lonicera japonica. https://www.biorxiv.org/content/10.1101/418319v1.full

59. Borse, S.P.; Singh, D.P.; Upadhyay, D.; Nivsarkar, M. Potential synergistic effects of quercetin with other phytoconstituents of Costus pictus (insulin plant) extract in the control of hyperglycemia and prevention of NSAID-induced gastroenteropathy in diabetic rats. Food Chem Toxicol 2018; 120, 48-61. 